\title{
Bedaquiline and delamanid for the treatment of multidrug-resistant tuberculosis: a multicentre cohort study in Korea
}

\author{
Cheon Tae Kim, Tae-Ok Kim², Hong-Joon Shin², Young Chun Ko ${ }^{3}$, \\ Yeong Hun $\mathrm{Choe}^{4}$, Hak-Ryul Kim ${ }^{5}$ and Yong-Soo Kwon ${ }^{2}$
}

Affiliations: ${ }^{1}$ Mokpo National TB Hospital, Mokpo, Republic of Korea. ${ }^{2}$ Dept of Internal Medicine, Chonnam National University Hospital, Gwangju, Republic of Korea. ${ }^{3}$ Dept of Internal Medicine, Kwangju Christian Hospital, Gwangju, Republic of Korea. ${ }^{4}$ Division of Respiratory Medicine and Allergy, Dept of Internal Medicine, Chonbuk National University Medical School, Jeonju, Republic of Korea. ${ }^{5}$ Dept of Internal Medicine, Institute of Wonkwang Medical Science, Wonkwang University School of Medicine, Iksan, Republic of Korea.

Correspondence: Yong-Soo Kwon, Dept of Internal Medicine, Chonnam National University Hospital, 42 Jebongro, Donggu, Gwangju 61469, Republic of Korea. E-mail: yskwonajnu.ac.kr

@ERSpublications

Bedaquiline and delamanid are effective and safe for MDR-TB treatment when combined with @WHO recommended regimens http://ow.ly/Xw8O30iqa0j

Cite this article as: Kim CT, Kim T-O, Shin H-J, et al. Bedaquiline and delamanid for the treatment of multidrug-resistant tuberculosis: a multicentre cohort study in Korea. Eur Respir J 2018; 51: 1702467 [https://doi.org/10.1183/13993003.02467-2017].

ABSTRACT Relatively little is known about the efficacy and safety of the programmatic use of bedaquiline and delamanid in multidrug-resistant tuberculosis (MDR-TB) treatment.

This study evaluated 61 patients with MDR-TB treated with bedaquiline $(n=39)$, delamanid $(n=11)$ or both, either sequentially $(n=10)$ or in coadministration $(n=1)$, for $>1$ month, combined with a World Health Organization-recommended regimen.

Of these, $49(80.3 \%)$ were male and $12(19.7 \%)$ were female. The median (interquartile range (IQR)) age was 53 (38.5-61.0) years. 42 (68.9\%) patients had fluoroquinolone-resistant MDR-TB and 16 (26.2\%) had extensively drug-resistant TB. The median (IQR) duration of treatment with bedaquiline and/or delamanid was 168 (166.5-196.5) days, with 33 (54.1\%) receiving linezolid for a median (IQR) of 673 (171-736) days. Of the 55 patients with positive sputum cultures at the start of bedaquiline and/or delamanid treatment, 39 (70.9\%) achieved sputum culture conversion within a median of 119 days. Treatment was halted in four patients (6.6\%) because of prolonged Fridericia's corrected QT interval.

Bedaquiline and delamanid were effective and safe for treating MDR-TB, with initial evidence of sequential administration of these two drugs as a viable treatment strategy for patients when an adequate treatment regimen cannot be constructed.

This article has supplementary material available from erj.ersjournals.com

Received: Nov 282017 | Accepted after revision: Feb 092018

Support statement: This study was supported by the National Research Foundation of Korea funded by the Korean Government (grant 2016R1D1A1B03931132). Funding information for this article has been deposited with the Crossref Funder Registry.

Conflict of interest: Y-S. Kwon reports receiving grants from the National Research Foundation of Korea during the conduct of the study.

Copyright OERS 2018 


\section{Introduction}

Multidrug-resistant tuberculosis (MDR-TB) is an important public health problem because of a lack of effective and safe anti-TB drugs and regimens. Treatment may entail the administration of many drugs over a long period of time, resulting in low treatment success rates and higher rates of adverse drug reactions (ADRs) [1-6]. Fluoroquinolones and second-line injectable drugs, such as kanamycin, amikacin and capreomycin, are considered crucial in the treatment of MDR-TB, but resistance to these agents may give rise to extensively drug-resistant (XDR)-TB. XDR-TB has become an emerging global health concern due the increased resistance to these effective anti-TB drugs [7-9].

Not only clinical trials but also clinical experiences have shown that bedaquiline and delamanid are promising new anti-TB drugs, with good efficacy and safety in the treatment of MDR-TB [10-26]. However, further data regarding their efficacy and safety, including the effects on QT prolongation, in patients with quinolone-resistant MDR-TB and XDR-TB are still required [27-31]. Moreover, sequential or coadministration treatment with bedaquiline and delamanid may improve treatment success in patients with quinolone-resistant MDR-TB and XDR-TB [21, 32-36]. This study therefore analysed the efficacy and safety of bedaquiline and/or delamanid in patients with MDR-TB treated under programmatic conditions.

\section{Methods}

\section{Study population}

This retrospective cohort study included patients with pulmonary MDR-TB treated between January 2015 and October 2017 at four tertiary referral hospitals and one TB-specific hospital in the Republic of Korea, each treating more than 300 patients with TB per year (figure 1). All enrolled patients underwent treatment with bedaquiline and/or delamanid for $>1$ month, in combination with a background regimen as recommended by the World Health Organization (WHO). TB was diagnosed based on the presence of Mycobacterium tuberculosis in sputum cultures. Sputum culture isolates of all patients were subjected to drug susceptibility tests (DSTs) for 15 anti-TB drugs on Löwenstein-Jensen medium. All DSTs were performed at the National Reference Laboratory of the Korean Institute of Tuberculosis (Osong, Republic of Korea) and Green Cross Laboratories (Yongin, Republic of Korea), using the proportion method. The tested drugs and their critical concentrations for resistance were: isoniazid $0.2 \mathrm{mg} \cdot \mathrm{L}^{-1}$, rifampin $40 \mathrm{mg} \cdot \mathrm{L}^{-1}$,

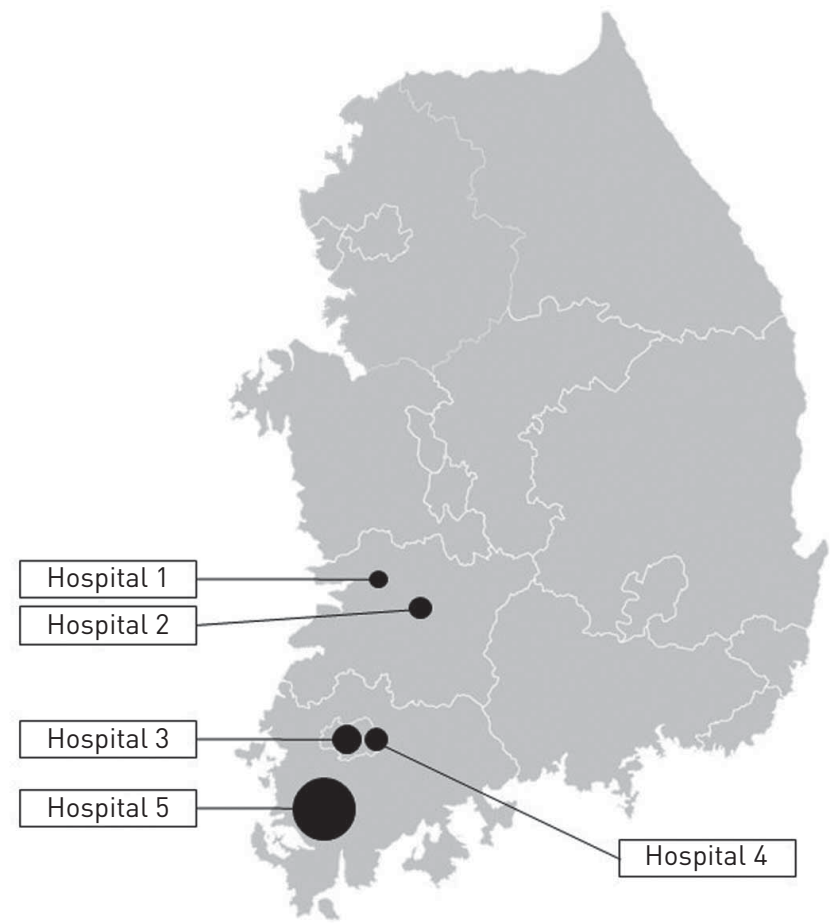

FIGURE 1 Distribution of five hospitals where patients with multidrug-resistant tuberculosis were treated with a regimen containing bedaquiline and/or delamanid in the Republic of Korea. The total number of patients was 61: three patients in Hospital 1 (lksan), five patients in Hospital 2 ( Jeonju), eight patients in Hospital 3 (Gwangju), five patients in Hospital 4 (Gwangju) and 40 patients in Hospital 5 (Mokpo). 
ethambutol $2 \mathrm{mg} \cdot \mathrm{L}^{-1}$, rifabutin $20 \mathrm{mg} \cdot \mathrm{L}^{-1}$, streptomycin $10 \mathrm{mg} \cdot \mathrm{L}^{-1}$, amikacin $40 \mathrm{mg} \cdot \mathrm{L}^{-1}$, kanamycin $40 \mathrm{mg} \cdot \mathrm{L}^{-1}$, capreomycin $40 \mathrm{mg} \cdot \mathrm{L}^{-1}$, ofloxacin $2 \mathrm{mg} \cdot \mathrm{L}^{-1}$, levofloxacin $2 \mathrm{mg} \cdot \mathrm{L}^{-1}$, moxifloxacin $2 \mathrm{mg} \cdot \mathrm{L}^{-1}$, prothionamide $40 \mathrm{mg} \cdot \mathrm{L}^{-1}$, cycloserine $30 \mathrm{mg} \cdot \mathrm{L}^{-1}$ and para-aminosalicylic acid $1 \mathrm{mg} \cdot \mathrm{L}^{-1}$. Pyrazinamide susceptibility was determined using the pyrazinamidase test. MDR-TB and XDR-TB were identified based on DST results of individual patients.

\section{Treatment and monitoring}

All patients underwent anti-TB treatment regimens as recommended by the WHO, tailored individually to each patient according to previous history of chemotherapy and DST results. Although the decision to start treatment with bedaquiline and/or delamanid was made by each attending physician, these new anti-TB drugs were prescribed only when an effective treatment regimen could not be provided, because of resistance to a drug, an ADR, poor tolerance or contraindication to any component of the combination regimen [19, 27-29]. The choice of bedaquiline and/or delamanid was based on the availability of these drugs and on patient condition. In Korea, the national expert committee reviewed all cases to be treated with bedaquiline and delamanid starting from September 2016 [19]. Accordingly, after this date, individual physicians were required to submit an application form to the national expert committee prior to prescribing the new anti-TB drugs. The national expert committee approved the use of new anti-TB drugs according to patient clinical status and/or when the patient met the criteria for the WHO guidelines.

All enrolled patients were monitored for drug regimen compliance and ADRs during treatment by specially trained nurses who participated in the Public-Private Mix project for TB control in Korea [37]. These nurses were responsible for direct drug administration during hospitalisation and provided support for self-administered drug treatment after discharge from the hospital. Laboratory tests, including sputum smear and culture, complete blood cell count, and liver and renal function tests, were performed every week during hospitalisation and at every monthly outpatient hospital visit during the treatment period. Additional laboratory tests were performed if patients manifested symptoms related to ADRs.

ECGs were recorded at baseline, and after 2, 4, 8, 12 and 24 weeks of treatment with bedaquiline and/or delamanid, as recommended by WHO guidelines [28]. The corrected QT interval was calculated as QT interval/RR interval using Fridericia's correction formula (QTcF) [38]. A significant QTcF prolongation was defined as an absolute value $>450 \mathrm{~ms}$ in males or $>470 \mathrm{~ms}$ in females, or as a $>60 \mathrm{~ms}$ increase from baseline [28].

Sputum culture conversion was defined as having at least two consecutive negative cultures taken at least 30 days apart in patients with a positive sputum specimen at baseline. The day of sputum collection for the first of two consecutive negative results was defined as the time of sputum culture conversion.

\section{Ethics statement}

The Institutional Review Board of Chonnam National University Hospital (Gwangju, Republic of Korea) approved the study protocol and provided permission for this study to be reviewed and published, including information obtained from patient records (CNUH-1017-168). Informed consent was waived because of the retrospective nature of the study, and patient information was anonymised and de-identified prior to analysis.

\section{Statistical analysis}

All data are reported as median (interquartile range (IQR)) or number (percentage). Continuous variables were analysed using the Mann-Whitney U-test for two groups or the Kruskal-Wallis test for three groups. Categorical variables were analysed using Fisher's exact test. Dunn's multiple comparison tests were used for post hoc correction to account for comparisons of three groups. All statistical analyses were performed using SPSS version 22.0 (IBM, Armonk, NY, USA), with $\mathrm{p}<0.05$ considered statistically significant.

\section{Results}

\section{Patient characteristics}

This study enrolled a total of 61 patients with pulmonary MDR-TB treated with bedaquiline and/or delamanid for $>1$ month. The median (IQR) age was 52 (38.5-61.0) years and 49 (80.3\%) patients were male. Of these, $39(64 \%), 11(18 \%)$ and $11(18 \%)$ were treated with bedaquiline, delamanid and both drugs, respectively. Patient baseline demographic and clinical characteristics are summarised in table 1. There were significant differences between the three groups in terms of the number of drugs to which the isolates were resistant and the percentage of patients resistant to fluoroquinolones, which was significantly higher among patients treated with both drugs than among those treated with bedaquiline alone, as revealed by Dunn's multiple comparison tests. 
TABLE 1 Baseline demographic and clinical characteristics of patients with multidrug-resistant tuberculosis (MDR-TB) treated with delamanid and/or bedaquiline

\begin{tabular}{|c|c|c|c|c|c|}
\hline & Total & Delamanid alone & Bedaquiline alone & Both drugs & p-value \\
\hline Subjects & 61 & 11 & 39 & 11 & \\
\hline Male & 49 (80.3) & $9(81.8)$ & $32(82.1)$ & $8(72.7)$ & 0.893 \\
\hline$\geqslant 45$ years & $42(68.9)$ & $7(63.6)$ & $27(69.2)$ & $8(72.7)$ & 0.926 \\
\hline$B M I \mathrm{~kg} \cdot \mathrm{m}^{-2}$ & $20.4(17.6-23.0)$ & $20.9(18.2-22.9)$ & $20.4(18.3-23.2)$ & $18.4(16.3-24.5)$ & 0.519 \\
\hline$<18.5 \mathrm{~kg} \cdot \mathrm{m}^{-2}$ & $19(31.1)$ & $3(27.3)$ & $10(25.6)$ & $6(54.5)$ & 0.246 \\
\hline Combined extrapulmonary TB & 2 (3.3) & $0(0)$ & $2(5.1)$ & $0(0)$ & 1.000 \\
\hline \multicolumn{6}{|l|}{ Comorbid conditions } \\
\hline Diabetes mellitus & 20 (32.8) & $4(36.4)$ & 13 (33.3) & 3 (27.3) & 1.000 \\
\hline Chronic lung disease & 7 (11.5) & $0(0)$ & 5 (12.8) & 2 (18.2) & 0.451 \\
\hline Chronic kidney disease & $3(4.9)$ & $1(9.1)$ & $2(5.1)$ & $0(0)$ & 0.741 \\
\hline Chronic liver disease & 13 (21.3) & $3(27.3)$ & $9(23.1)$ & $1(9.1)$ & 0.666 \\
\hline MDR-TB with SLID resistance & $19(31.1)$ & $3(27.3)$ & $12(30.8)$ & $4(36.4)$ & 0.925 \\
\hline MDR-TB with fluoroquinolone resistance & $42(68.9)$ & $9(81.8)$ & $22(56.4)^{\#}$ & $11(100)^{\#}$ & 0.009 \\
\hline XDR-TB & $16(26.2)$ & $3(27.3)$ & $9(23.1)$ & $4(36.4)$ & 0.707 \\
\hline Positive sputum smear & $34(55.7)$ & $6(54.5)$ & $21(53.8)$ & $7(63.6)$ & 0.931 \\
\hline Cavity (or cavities) on chest radiography & $30(49.2)$ & $5(45.5)$ & 20 (51.3) & $5(45.5)$ & 1.000 \\
\hline Bilateral disease & $45(73.8)$ & $7(63.6)$ & $27(69.2)$ & $11(100)$ & 0.075 \\
\hline \multicolumn{6}{|l|}{ Baseline laboratory tests } \\
\hline Haemoglobin $\mathrm{g} \cdot \mathrm{dL}^{-1}$ & $13.2(11.7-14.2)$ & $12.9(11.7-14.6)$ & $12.9(11.5-14.2)$ & $14.0(11.8-14.0)$ & 0.846 \\
\hline Albumin $\mathrm{g} \cdot \mathrm{L}^{-1}$ & $3.9(3.6-4.3)$ & $3.7(3.6-4.1)$ & $3.9(3.5-4.2)$ & $4.3(3.9-4.5)$ & 0.065 \\
\hline AST IU.L $L^{-1}$ & $24.0(18.5-32.5)$ & $28.0(23.0-35.0)$ & $24.0(18.0-35.0)$ & $23.0(17.0-27.0)$ & 0.288 \\
\hline$A L T I U \cdot L^{-1}$ & $14.0(8.0-21.5)$ & $18.0(9.0-21.0)$ & $13.0(8.0-19.0)$ & $15.0(8.0-24.0)$ & 0.629 \\
\hline Total bilirubin $\mathrm{g} \cdot \mathrm{dL}^{-1}$ & $0.5(0.4-0.7)$ & $0.4(0.3-0.7)$ & $0.5(0.4-0.8)$ & $0.6(0.4-0.7)$ & 0.749 \\
\hline Baseline QTcF ms & $441.0(427.0-461.0)$ & $441.0(427.0-466.0)$ & $438.0(427.0-460.0)$ & $449.0(423.0-463.0)$ & 0.660 \\
\hline
\end{tabular}

Data are presented as $\mathrm{n}, \mathrm{n}(\%)$ or median (interquartile range). BMI: body mass index; SLID: second-line injectable drug; XDR: extensively drug-resistant; AST: aspartate aminotransferase; ALT: alanine aminotransferase; QTcF: Fridericia's corrected QT interval. Dunn's post hoc test was performed when the result of the Kruskal-Wallis test was significant: ${ }^{\#}: p<0.05$.

Isolates from 49 (80.3\%), $44(71.5 \%)$ and 28 (45.9\%) patients were resistant to ethambutol, pyrazinamide and streptomycin, respectively. Assessment of resistance to second-line injectable drugs and fluoroquinolones showed that 19 (31.1\%) isolates were resistant to kanamycin and 42 (68.9\%) isolates were resistant to ofloxacin (table 2).

Treatment

Of the 11 patients treated with both bedaquiline and delamanid, 10 were treated sequentially and one was treated with coadministration. Of the 10 patients treated sequentially, nine received bedaquiline followed by delamanid at a median (IQR) interval of 71 (56-78) days and one received delamanid followed by bedaquiline 1 day later. The median (IQR) duration of treatment with bedaquiline and/or delamanid was 168 (166.5-196.5) days, with no difference between the groups treated with delamanid and bedaquiline alone. However, length of treatment was significantly longer in patients treated with both drugs than in those treated with one or the other (table 3). 50 patients (82.0\%) completed 6 months of treatment, including nine $(81.8 \%)$ treated with delamanid alone, $34(87.2 \%)$ treated with bedaquiline alone and seven (63.6\%) treated with both drugs. Of 55 patients who completed 6 months of treatment, eight patients were treated with bedaquiline $(n=6)$ or delamanid $(n=2)$ for $>190$ days. The median (IQR) duration of bedaquiline therapy was 323 (222-394) days; the duration of delamanid therapy was 210 and 237 days, respectively. Of the 11 patients who did not complete 6 months of treatment, four were administered new anti-TB drugs at the end of this study period, while seven stopped treatment: five discontinued the drugs because of ADRs, one patient died of an underlying malignancy after treatment with bedaquiline for 126 days and one patient discontinued treatment due to termination of health insurance cover after 119 days of bedaquiline treatment. 
TABLE 2 Drug resistance rates in patients with multidrug-resistant tuberculosis

$\begin{array}{lr}\text { Isoniazid } & 61(100) \\ \text { Rifampin } & 61(100) \\ \text { Rifabutin } & 49(80.3) \\ \text { Ethambutol } & 49(80.3) \\ \text { Pyrazinamide } & 43(70.5) \\ \text { Ofloxacin } & 42(68.9) \\ \text { Levofloxacin } & 41(67.2) \\ \text { Moxifloxacin } & 38(62.3) \\ \text { Prothionamide } & 30(49.2) \\ \text { Streptomycin } & 28(45.9) \\ \text { Para-aminosalicylic acid } & 20(32.8) \\ \text { Kanamycin } & 19(31.1) \\ \text { Cycloserine } & 17(27.9) \\ \text { Amikacin } & 16(26.2) \\ \text { Capreomycin lor viomycin) } & 12(19.7)\end{array}$

Data are presented as $\mathrm{n}(\%)$.

There were significant differences in the percentage of patients treated with fluoroquinolones, linezolid and clofazimine in the three treatment groups. Even after post-test correction using Dunn's multiple comparison tests, the frequency of fluoroquinolone use was significantly lower in the group treated with both drugs than in the group treated with bedaquiline alone. In contrast, the frequency of clofazimine use was significantly higher in the group treated with both drugs than in the group treated with bedaquiline alone. Moreover, the duration of treatment with bedaquiline and/or delamanid was significantly longer in the group treated with both drugs than in the groups treated with bedaquiline or delamanid alone (table 3). Treatment with other anti-TB drugs did not differ significantly in the three groups, except that clarithromycin and amoxicillin-clavulanic acid frequencies were higher in the group treated with both bedaquiline and delamanid than in the monotherapy groups (supplementary table S1).

TABLE 3 Treatment, outcomes and QT prolongation of patients with multidrug-resistant tuberculosis treated with delamanid and/or bedaquiline

\begin{tabular}{|c|c|c|c|c|c|}
\hline Treatment & Total & Delamanid alone & Bedaquiline alone & Both drugs & p-value \\
\hline $\begin{array}{l}\text { Drugs administered, including delamanid } \\
\text { and/or bedaquiline } \mathrm{n}\end{array}$ & $5.0(4.0-6.0)$ & $5.0(4.0-6.0)$ & $5.0(4.0-6.0)$ & $5.0(3.0-6.0)$ & 0.728 \\
\hline Treatment with any injectable drug & 39 (63.9) & $6(54.5)$ & $25(64.1)$ & $8(72.7)$ & 0.688 \\
\hline Duration of injectable drug treatment days & $224.0(188.0-280.0)$ & $229.0(168.5-268.3)$ & $224.0(198.5-297.5)$ & $230.0(166.3-537.3)$ & 0.863 \\
\hline Treatment with any fluoroquinolone & $37(60.7)$ & $7(63.6)$ & $27(69.2)^{\pi}$ & $3(27.3)^{\pi}$ & 0.043 \\
\hline Duration of linezolid treatment days & $673.0(171.0-736.0)$ & $154.5(49.0-413.3)$ & $617.0(136.0-759.0)$ & $729.5(673.0-874.5)$ & 0.094 \\
\hline $\begin{array}{l}\text { Duration of delamanid and/or bedaquiline } \\
\text { treatment days }\end{array}$ & $168.0(166.5-196.5)$ & $168.0(167.0-176.0)^{+}$ & $168.0(165.0-182.0)^{\pi}$ & $341.0(230.0-375.0)^{1,+}$ & 0.001 \\
\hline Combined surgery $\#$ & $5(8.5)$ & $2(18.2)$ & $1(2.7)$ & $2(18.2)$ & 0.102 \\
\hline Culture conversion & $39 / 55(70.9)$ & $8 / 8(100)$ & $24 / 36(66.7)$ & $7 / 11(63.6)$ & 0.160 \\
\hline Time to culture conversion days & $119.0(52.5-198.5)$ & $122.0(53.0-145.3)^{+}$ & $84.0(35.5-174.0)^{\pi}$ & $307.5(235.0-346.5)^{\uparrow,+}$ & $<0.001$ \\
\hline
\end{tabular}

Data are presented as $\mathrm{n}$, median (interquartile range) or $\mathrm{n}(\%)$, unless otherwise stated. QTcF: Fridericia's corrected QT interval. Dunn's post hoc test was performed when the result of the Kruskal-Wallis test was significant: ${ }^{\#}$ : $p>0.05$ among groups; ${ }^{\text {ๆ }}$ : $p<0.05$ bedaquiline versus both drugs; ${ }^{+}: p<0.05$ delamanid versus both drugs. 


\section{Efficacy and safety of new drugs}

Of the 61 patients with MDR-TB, 55 (90.2\%) had positive sputum cultures at the time of starting treatment with bedaquiline and/or delamanid. Of these, 39 (70.9\%) achieved culture conversion, although the percentage of patients achieving culture conversion did not differ among the three treatment groups $(\mathrm{p}=0.160)$ (table 3$)$. We evaluated factors potentially associated with culture conversion, including age $\geqslant 45$ years, male sex, low body mass index, previous TB treatment history, smear positivity, the presence of a cavity (or cavities) on chest radiography, XDR-TB, use of linezolid, use of new anti-TB drugs such as bedaquiline and/or delamanid, and treatment duration of new anti-TB drugs. No significant associations were found (supplementary table S2).

Regarding the time to culture conversion from the start of treatment with bedaquiline and/or delamanid of patients administered both drugs, all seven patients who achieved culture conversion received sequential treatment and the median (IQR) time to culture conversion was 307.5 (235.0-346.5) days, which was significantly longer than that in the groups treated with bedaquiline $(\mathrm{p}<0.001)$ or delamanid $(\mathrm{p}=0.004)$ alone, even after post hoc analysis (table 3).

28 patients experienced ADRs, resulting in discontinuation of anti-TB drugs. Eight patients experienced optic neuropathy, caused by linezolid in five patients and ethambutol in three patients; seven patients experienced peripheral neuropathy caused by linezolid; and in four we observed prolonged QTcF caused by bedaquiline and/or delamanid. Furthermore, two patients developed gastrointestinal problems caused by para-aminosalicylic acid and clarithromycin, respectively; two patients developed anaemia caused by linezolid; two patients developed azotaemia caused by kanamycin and streptomycin, respectively; one patient experienced alopecia caused by delamanid; one patient experienced skin eruptions and one patient developed hepatotoxicity of unknown cause after stopping delamanid and bedaquiline. Five patients (8.2\%) experienced ADRs that resulted in stopping delamanid and/or bedaquiline, including four with a significantly prolonged QTcF. Of these four patients, one was treated with delamanid and bedaquiline sequentially, one with both drugs simultaneously, one with bedaquiline, and one with delamanid (table 4). The frequency of stopping delamanid and/or bedaquiline due to a prolonged QTcF was higher in patients being treated with both drugs than in the other groups, although the differences were not statistically significant (table 3). QTcF became normalised in all four of these patients who stopped delamanid and/or bedaquiline due to prolonged QTcF, and these drugs were not re-introduced. A fifth patient stopped delamanid on day 137 due to severe alopecia, which developed 1 month after commencing treatment and progressively worsened.

\section{Discussion}

This cohort study found that treatment of patients with MDR-TB with the new anti-TB drugs delamanid and bedaquiline, individually or sequentially, showed good efficacy and safety in combination with the WHO-recommended background regimens. Previous clinical trials showed that treatment with bedaquiline for 24 weeks resulted in sputum culture conversion in $79-81 \%$ of patients at week 24 and in $62-72 \%$ at week $120[10,11,14]$. Moreover, the combination of an optimised background regimen with delamanid

\begin{tabular}{|c|c|c|c|c|c|c|c|c|c|}
\hline Patient & Group & $\begin{array}{c}\text { Age } \\
\text { years }\end{array}$ & Sex & $\begin{array}{c}\text { Drug } \\
\text { resistance }\end{array}$ & $\begin{array}{c}\text { Duration of } \\
\text { delamanid } \\
\text { treatment } \\
\text { days }\end{array}$ & $\begin{array}{c}\text { Duration of } \\
\text { bedaquiline } \\
\text { treatment } \\
\text { days }\end{array}$ & $\begin{array}{l}\text { Baseline } \\
\text { QTcF ms }\end{array}$ & $\begin{array}{c}\text { Maximum } \\
\text { QTcF ms }\end{array}$ & $\begin{array}{c}\text { Concomitant } \\
\text { drugs with an } \\
\text { increased risk of } \\
\text { QTcF } \\
\text { prolongation }\end{array}$ \\
\hline 2 & Delamanid & 72 & Male & XDR-TB & 103 & & 484 & 574 & No \\
\hline 3 & Bedaquiline & 85 & Female & MDR-TB & & 145 & 446 & 521 & $\begin{array}{l}\text { Moxifloxacin, } \\
\text { clarithromycin }\end{array}$ \\
\hline
\end{tabular}

MDR: multidrug-resistant; TB: tuberculosis; XDR: extensively drug-resistant. 
for 6-8 months resulted in a sputum culture conversion rate of $65 \%$ at 8 weeks and a favourable outcome rate of $75 \%$ after 24 months $[12,13]$. In a recent large observational cohort study under different settings, except for the experimental conditions, bedaquiline-containing regimens achieved sputum culture conversion rates $>90 \%$ at the end of treatment, even with a high proportion of fluoroquinolone-resistant TB (64.5\%) and XDR-TB (45.6\%) [17]. High culture conversion rates have also been reported in other recent cohort studies of new anti-TB drugs in patients with MDR-TB [15, 16, 18-26]. In this study, an overall culture conversion rate of $71 \%$ was achieved, despite $69 \%$ of patients having fluoroquinolone-resistant MDR-TB and 26\% having XDR-TB. This was relatively high compared with the percentages in previous clinical trials, but not as high as those in observational cohort studies. This difference may be caused by differences in patient conditions, clinical settings and regimens.

In comparing bedaquiline and delamanid, we found that all patients treated with delamanid achieved culture conversion, compared with $67 \%$ of patients treated with bedaquiline who achieved sputum culture conversion, although the difference was not statistically significant. Owing to the small number of patients treated with delamanid, we could not explain this difference in culture conversion rates. However, patients treated with delamanid tended to be younger and have higher rates of combined surgery, and these factors may account for the differences in culture conversion. Furthermore, over half of patients (51.3\%) treated with bedaquiline were prescribed the drug in 2015 , whereas the majority of patients $(71.4 \%)$ treated with delamanid were prescribed the drug in 2016. Physicians may have better knowledge about treating patients with MDR-TB with new anti-TB drugs, especially when they prescribe delamanid, and this may explain the differences in culture conversion between the two drugs.

One of the most important safety issues associated with these new anti-TB drugs is drug-induced QTcF prolongation, which is usually asymptomatic and may cause fatal ventricular tachyarrhythmia [27-30]. The new anti-TB drugs, as well as interactions between these drugs and other anti-TB drugs, can cause QTcF prolongation and most clinical trials had strict criteria in selecting anti-TB drugs for inclusion in background regimens [10-14]. A recent systematic analysis of cardiac safety with bedaquiline treatment involving 1256 patients, including controlled clinical trials and cohort studies, showed that QTc was $>500 \mathrm{~ms}$ in $3.2 \%$ of patients and $0.6 \%$ of patients discontinued treatment due to QTc prolongation [31]. Although Pontali et al. [31] concluded that bedaquiline is a relatively safe drug, this conclusion may be limited due to lack of information regarding cardiac safety and no standardised way to report cardiac safety in most studies [31]. Therefore, more information about cardiac safety of new anti-TB drugs, including within various regimens and settings, is required. However, in our study, 69\% of patients experienced significant QTcF prolongation and only 7\% had to discontinue delamanid and/or bedaquiline because of QTcF prolongation, and none experienced drug-induced fatal arrhythmia. This finding was comparable to recent cohort studies testing new anti-TB drugs in patients with MDR-TB [15-19, 23, 24, 26]. Among factors related to QTcF prolongation, coadministration of drugs may increase the risk of QTcF prolongation. In our study, patients treated with clofazimine (28.6\% versus $0 \% ; \mathrm{p}=0.012)$ and clarithromycin $(33.3 \%$ versus $0 \% ; \mathrm{p}=0.003)$ experienced a significantly higher rate of significant $\mathrm{QTcF}$ prolongation. Use of both drugs together should also be considered as another factor in relation to QTcF prolongation. Of the four patients who discontinued delamanid and/or bedaquiline because of QTcF prolongation in this study, two were treated with both drugs.

Although nine $(82 \%)$ of the 11 patients treated with both drugs were treated sequentially with bedaquiline followed by delamanid at a median interval of 71 days, treatment was halted only in one patient treated initially with delamanid, followed by bedaquiline. This finding is interesting, considering the long half-life of bedaquiline and the potential risk of ADRs when delamanid is used subsequently before a washout period of bedaquiline. Furthermore, the WHO suggested a delamanid-bedaquiline sequence and a 5-day washout period of delamanid is recommended before using bedaquiline due to the short half-life of delamanid [28]. However, in Korea, bedaquiline was first approved in April 2014 and delamanid was approved in October 2015. All patients treated sequentially in this study were commenced on bedaquiline before approval of delamanid in Korea, except for one patient undergoing a delamanid-bedaquiline sequence. Patients undergoing the bedaquiline-delamanid sequence had no choice in deciding which of the new anti-TB drugs was administered first. Although bedaquiline has a long half-life, the area under the concentration curve declined rapidly within 2-3 weeks of treatment, followed by a slow elimination [39]. Therefore, the blood concentration of bedaquiline, when patients received delamanid, may not sufficiently influence QT prolongation. Moreover, as the recent cases of coadministration of the two drugs showed no significant adverse reactions, bedaquiline-delamanid sequential therapy, regardless of the time interval between the two drugs, may not be an important factor in serious adverse reactions.

Few studies to date have assessed combinations of delamanid and bedaquiline with WHO-recommended background regimens [23, 25, 32-35]. Although most of these studies reported a favourable response, there are still concerns about QTcF prolongation, suggesting the need for additional safety data. One patient in 
our study was administered both new anti-TB drugs and developed a significant QTcF prolongation, of $521 \mathrm{~ms}$, at 41 days, requiring discontinuation of both delamanid and bedaquiline. This patient failed sputum culture conversion through the end of the study period. These findings suggest that care should be taken in selecting patients for coadministration of both new anti-TB drugs and that these patients should be carefully monitored for QTcF prolongation.

In the sequential use of both new anti-TB drugs, few cases have been reported and without detailed information regarding efficacy and safety [35]. All 10 of our patients treated sequentially had fluoroquinolone-resistant MDR-TB, with seven achieving culture conversion, which may be higher than that in a previous meta-analysis of individual patient data and a large cohort study in Korea for MDR-TB treatment without using new anti-TB drugs $[3,40]$. The time to culture conversion in sequential use of both new anti-TB drugs was significantly longer than those in the groups treated with bedaquiline or delamanid alone and may also be caused by higher drug resistance, as shown in a previous study in patients requiring prolonged use of bedaquiline [18]. However, one of these patients experienced significant QTcF prolongation which required discontinuation of both these drugs. Patients treated sequentially with these drugs should be carefully monitored for QTcF prolongation.

Regarding treatment duration of the new anti-TB drugs, eight patients received prolonged ( $>190$ days) treatment of bedaquiline or delamanid in this study. The WHO recommends 6 months of treatment for individual drugs, although in some cases this may not be sufficient and a longer period may be required. Good outcomes with prolonged use of bedaquiline have recently been reported, although further data are needed to clarify this issue [18].

One patient (a 55-year-old male) in this study discontinued bedaquiline treatment due to the termination of health insurance cover and failed to achieve negative sputum conversion until the end of the study period. This could be a public health risk in terms of transmission of highly resistant TB. The decision to discontinue treatment occurred prior to the introduction of the national expert committee that approves new anti-TB drugs after a careful review of individual cases in Korea. Another important issue is the possibility of drug resistance to the new anti-TB drugs in the 16 patients who did not achieve culture conversion at the end of the study period. We could not evaluate drug resistance and this is a limitation of the present study; this could be of great concern in the TB community. Therefore, appropriate regulatory efforts in approving the prescription of new anti-TB drugs, as well as prudent decision making by physicians when prescribing these drugs, are required to increase the cure rate of MDR-TB and prevent the development of additional drug resistance to these valuable new anti-TB drugs during treatment.

This study has several limitations due to its retrospective design. First, decisions about drug treatment were made by individual attending physicians. Therefore, patients had different treatment regimens. Second, there may have been a selection bias among enrolled patients. Owing to the high prices of delamanid and bedaquiline, relatively young and economically well-off patients would likely be candidates for their use. In Korea, however, the costs of all anti-TB drugs, including new drugs, are paid by government-controlled health insurance. Therefore, the decision to treat patients with these new anti-TB drugs may be unrelated to age or economic status. Third, the low number of patients treated with delamanid prevented statistical comparisons of the efficacy and safety of bedaquiline and delamanid. Bedaquiline was introduced earlier than delamanid, including for compassionate use, resulting in the higher number of patients treated with bedaquiline than with delamanid in this study. Fourth, the efficacy and safety of the re-introduction of new anti-TB drugs after discontinuation due to ADRs could not be evaluated. The new anti-TB drugs in this study were not administered again once discontinued. Considering the limited number of effective drugs in MDR-TB treatment, re-introduction after discontinuation of new anti-TB drugs could be considered. Fifth, the distribution of treatment groups according to study periods and sites was not even. Bedaquiline was mostly prescribed in the early study period (2015) and over half of delamanid was prescribed in the mid study period (2016). In some study sites there were no cases of delamanid prescription and most prescriptions of both drugs (91\%) were in a single centre. However, there were no significant differences in culture conversion according to study periods and sites.

In conclusion, treatment of patients with MDR-TB with bedaquiline and/or delamanid, including their sequential use, was generally effective and well tolerated. However, patients treated with both drugs, whether simultaneously or sequentially, should be carefully monitored for QTcF prolongation.

\section{Acknowledgements}

The authors thank Min-Ho Shin (Chonnam National University Medical School, Gwangju, Republic of Korea) for supporting the statistical analysis.

Author contributions: Y-S. Kwon was the lead researcher, and was responsible for study design, data analysis and manuscript preparation. All authors contributed to the generation and analysis of the data, and wrote the manuscript. All authors read and approved the final version of the manuscript. 


\section{References}

1 World Health Organization. Global tuberculosis report 2017. WHO/HTM/TB/2017.23. 2017. http://apps.who.int/ iris/bitstream/10665/259366/1/9789241565516-eng.pdf Date last accessed: December 1, 2017.

2 Ahuja SD, Ashkin D, Avendano M, et al. Multidrug resistant pulmonary tuberculosis treatment regimens and patient outcomes: an individual patient data meta-analysis of 9,153 patients. PLoS Med 2012; 9: e1001300.

3 Kim DH, Kim HJ, Park SK, et al. Treatment outcomes and long-term survival in patients with extensively drug-resistant tuberculosis. Am J Respir Crit Care Med 2008; 178: 1075-1082.

4 Kwon YS, Kim YH, Suh GY, et al. Treatment outcomes for HIV-uninfected patients with multidrug-resistant and extensively drug-resistant tuberculosis. Clin Infect Dis 2008; 47: 496-502.

5 Johnston JC, Shahidi NC, Sadatsafavi M, et al. Treatment outcomes of multidrug-resistant tuberculosis: a systematic review and meta-analysis. PLoS One 2009; 4: e6914.

6 Orenstein EW, Basu S, Shah NS, et al. Treatment outcomes among patients with multidrug-resistant tuberculosis: systematic review and meta-analysis. Lancet Infect Dis 2009; 9: 153-161.

7 Jacobson KR, Tierney DB, Jeon CY, et al. Treatment outcomes among patients with extensively drug-resistant tuberculosis: systematic review and meta-analysis. Clin Infect Dis 2010; 51: 6-14.

8 Migliori GB, Sotgiu G, Gandhi NR, et al. Drug resistance beyond extensively drug-resistant tuberculosis: individual patient data meta-analysis. Eur Respir J 2013; 42: 169-179.

9 Pietersen E, Ignatius E, Streicher EM, et al. Long-term outcomes of patients with extensively drug-resistant tuberculosis in South Africa: a cohort study. Lancet 2014; 383: 1230-1239.

10 Diacon AH, Pym A, Grobusch M, et al. The diarylquinoline TMC207 for multidrug-resistant tuberculosis. N Engl J Med 2009; 360: 2397-2405.

11 Diacon AH, Pym A, Grobusch MP, et al. Multidrug-resistant tuberculosis and culture conversion with bedaquiline. N Engl J Med 2014; 371: 723-732.

12 Gler MT, Skripconoka V, Sanchez-Garavito E, et al. Delamanid for multidrug-resistant pulmonary tuberculosis. $N$ Engl J Med 2012; 366: 2151-2160.

13 Skripconoka V, Danilovits M, Pehme L, et al. Delamanid improves outcomes and reduces mortality in multidrug-resistant tuberculosis. Eur Respir J 2013; 41: 1393-1400.

14 Pym AS, Diacon AH, Tang SJ, et al. Bedaquiline in the treatment of multidrug- and extensively drug-resistant tuberculosis. Eur Respir J 2016; 47: 564-574.

15 Guglielmetti L, Le Du D, Jachym M, et al. Compassionate use of bedaquiline for the treatment of multidrug-resistant and extensively drug-resistant tuberculosis: interim analysis of a French cohort. Clin Infect Dis 2015; 60: 188-194.

16 Ndjeka N, Conradie F, Schnippel K, et al. Treatment of drug-resistant tuberculosis with bedaquiline in a high HIV prevalence setting: an interim cohort analysis. Int J Tuberc Lung Dis 2015; 19: 979-985.

17 Borisov SE, Dheda K, Enwerem M, et al. Effectiveness and safety of bedaquiline-containing regimens in the treatment of MDR- and XDR-TB: a multicentre study. Eur Respir J 2017; 49: 1700387.

18 Guglielmetti L, Jaspard M, Le Du D, et al. Long-term outcome and safety of prolonged bedaquiline treatment for multidrug-resistant tuberculosis. Eur Respir J 2017; 49: 1601799.

19 Mok J, Kang H, Hwang SH, et al. Interim outcomes of delamanid for the treatment of MDR- and XDR-TB in South Korea. J Antimicrob Chemother 2018; 73: 503-508.

20 Olaru ID, Heyckendorf J, Andres S, et al. Bedaquiline-based treatment regimen for multidrug-resistant tuberculosis. Eur Respir J 2017; 49: 1700742.

21 Pontali E, Sotgiu G, D’Ambrosio L, et al. Bedaquiline and multidrug-resistant tuberculosis: a systematic and critical analysis of the evidence. Eur Respir J 2016; 47: 394-402.

22 Pontali E, D'Ambrosio L, Centis R, et al. Multidrug-resistant tuberculosis and beyond: an updated analysis of the current evidence on bedaquiline. Eur Respir J 2017; 49: 1700146.

23 Hafkin J, Hittel N, Martin A, et al. Early outcomes in MDR-TB and XDR-TB patients treated with delamanid under compassionate use. Eur Respir J 2017; 50: 1700311.

24 Hewison C, Ferlazzo G, Avaliani Z, et al. Six-month response to delamanid treatment in MDR TB patients. Emerg Infect Dis 2017; 23: 1746-1748.

25 Kuksa L, Barkane L, Hittel N, et al. Final treatment outcomes of multidrug- and extensively drug-resistant tuberculosis patients in Latvia receiving delamanid-containing regimens. Eur Respir J 2017; 50: 1701105.

26 Udwadia ZF, Ganatra S, Mullerpattan JB. Compassionate use of bedaquiline in highly drug-resistant tuberculosis patients in Mumbai, India. Eur Respir J 2017; 49: 1601699.

27 World Health Organization. The use of bedaquiline in the treatment of multidrug-resistant tuberculosis. Interim policy guidance. WHO/HTM/TB/2013.6. 2013. http://apps.who.int/iris/bitstream/10665/84879/1/9789241505482_ eng.pdf Date last accessed: December 1, 2017.

28 World Health Organization. Companion handbook to the WHO guidelines for the programmatic management of drug-resistant tuberculosis. WHO/HTM/TB/2014.11. 2014. http://apps.who.int/iris/bitstream/10665/130918/1/ 9789241548809_eng.pdf Date last accessed: December 1, 2017.

29 World Health Organization. The use of delamanid in the treatment of multidrug-resistant tuberculosis. Interim policy guidance. WHO/HTM/TB2014.23. 2014. http://apps.who.int/iris/bitstream/10665/137334/1/WHO_HTM_ TB_2014.23_eng.pdf Date last accessed: December 1, 2017.

30 Roden DM. Drug-induced prolongation of the QT interval. N Engl J Med 2004; 350: 1013-1022.

31 Pontali E, Sotgiu G, Tiberi S, et al. Cardiac safety of bedaquiline: a systematic and critical analysis of the evidence. Eur Respir J 2017; 50: 1701462.

32 Lachatre M, Rioux C, Le Du D, et al. Bedaquiline plus delamanid for XDR tuberculosis. Lancet Infect Dis 2016; 16: 294.

33 Tadolini M, Lingtsang RD, Tiberi S, et al. First case of extensively drug-resistant tuberculosis treated with both delamanid and bedaquiline. Eur Respir J 2016; 48: 935-938.

34 Tadolini M, Lingtsang RD, Tiberi $\mathrm{S}$, et al. Cardiac safety of extensively drug-resistant tuberculosis regimens including bedaquiline, delamanid and clofazimine. Eur Respir J 2016; 48: 1527-1529.

35 Maryandyshev A, Pontali E, Tiberi S, et al. Bedaquiline and delamanid combination treatment of 5 patients with pulmonary extensively drug-resistant tuberculosis. Emerg Infect Dis 2017; 23: 1718-1721. 
36 Migliori GB, Pontali E, Sotgiu G, et al. Combined use of delamanid and bedaquiline to treat multidrug-resistant and extensively drug-resistant tuberculosis: a systematic review. Int J Mol Sci 2017; 18: 341.

37 Park CK, Shin HJ, Kim YI, et al. Predictors of default from treatment for tuberculosis: a single center case-control study in Korea. J Korean Med Sci 2016; 31: 254-260.

38 Fridericia LS. The duration of systole in an electrocardiogram in normal humans and in patients with heart disease. 1920. Ann Noninvasive Electrocardiol 2003; 8: 343-351.

39 McLeay SC, Vis P, van Heeswijk RP, et al. Population pharmacokinetics of bedaquiline (TMC207), a novel antituberculosis drug. Antimicrob Agents Chemother 2014; 58: 5315-5324.

40 Falzon D, Gandhi N, Migliori GB, et al. Resistance to fluoroquinolones and second-line injectable drugs: impact on multidrug-resistant TB outcomes. Eur Respir J 2013; 42: 156-168. 